\title{
ON THE STRENGTH OF SAND
}

\author{
B. C. RENNIE
}

(rec. 18 Nov. 1957; revised 16 Dec. 1958)

\section{Introduction}

An aggregate consisting of a large number of small, equal, hard spheres might be expected to behave like ordinary sand; in this article therefore an attempt is made to find the porperties that will be exhibited by this model, and in particular to find what stresses the sand will withstand without collapsing.

Consider a region of the sand that is arranged in the 'lattice packing' that is believed to be the closest possible packing of spheres. Such a latticepacked region is not isotropic as regards its strength, so we suppose that the sand as a whole will withstand some particular stress if all possible orientations of the lattice-packed region will withstand it. In other words we suppose that a large region of sand contains a large number of latticepacked regions oriented at random and that a uniform stress applied to the whole region remains uniform over all the small regions and if it causes the collapse of one of the small regions it will cause the collapse of the sand as a whole.

We first solve the problem for smooth spheres, and then in Section II for a small amount of friction we find the solution to first order in the coefficient of friction. Finally in Section III we discuss empirical results and the remarkable firmness of a sandy beach a little way up from the edge of the sea.

\section{The Case of No Friction}

We suppose that the sand is arranged in the lattice packing given by the centres of the spheres at the points with Cartesian coordinates Pnd where $\boldsymbol{n}$ is any column of three integers, $d$ is the diamater of a sand grain and $\boldsymbol{P}$ is the matrix:-

$$
\boldsymbol{P}=\left(\begin{array}{rrr}
1 & \frac{1}{2} & \frac{1}{2} \\
0 & \sqrt{\frac{1}{2}} & \sqrt{\frac{1}{2}} \\
0 & -\frac{1}{2} & \frac{1}{2}
\end{array}\right)
$$


This formula gives the distance from the origin to the centre of any other sphere (or the distance between any two spheres) as

$$
\mathrm{d} \sqrt{\boldsymbol{n}^{\prime} \boldsymbol{P}^{\prime} \boldsymbol{P n}} \text { or } \mathrm{d} \sqrt{n_{1}^{2}+n_{2}^{2}+n_{3}^{2}+n_{1} n_{2}+n_{2} n_{3}+n_{3} n_{1}} .
$$

This expression reaches its minimum value of $d$ at twelve points, given by the row $\boldsymbol{n}^{\prime}$ equal to:-

$$
(1,0,0),(0,1,0),(0,0,1),(0,1,-1),(1,0,-1) \text { and }(1,-1,0)
$$

and their negatives, so that each sphere touches twelve others, diametrically opposite in pairs.

Now suppose that the sand is subjected to a uniform small strain $\boldsymbol{S}$, so that the grain of sand originally at $\boldsymbol{x}$ is moved to $(\mathbf{1}+\boldsymbol{S}) \boldsymbol{x}$. The square of the distance of a typical grain from the centre will change from $\boldsymbol{n}^{\prime} \boldsymbol{P}^{\prime} \boldsymbol{P} \boldsymbol{n} \mathrm{d}^{2}$ to $\boldsymbol{n}^{\prime} \boldsymbol{P}^{\prime}\left(\mathbf{1}+\boldsymbol{S}^{\prime}\right)(\mathbf{1}+\boldsymbol{S}) \boldsymbol{P n d ^ { 2 }}$ Taking $\boldsymbol{S}$ to be symmetric, the change in this square will be $2 \boldsymbol{n}^{\prime} \boldsymbol{P}^{\prime} \boldsymbol{S} \boldsymbol{P} \boldsymbol{n} \mathrm{d}^{2}$ to first order in $\boldsymbol{S}$. For any of the twelve grains touching the central one this quantity must be positive, so that six inequalities of the form $\boldsymbol{n}^{\prime} \boldsymbol{P}^{\prime} \boldsymbol{S P n} \geqq 0$ must hold for a strain $\boldsymbol{S}$ to be geometrically possible, and these conditions are also clearly sufficient.

The conditions corresponding to each of the six values of $\boldsymbol{n}^{\prime}$ are:-

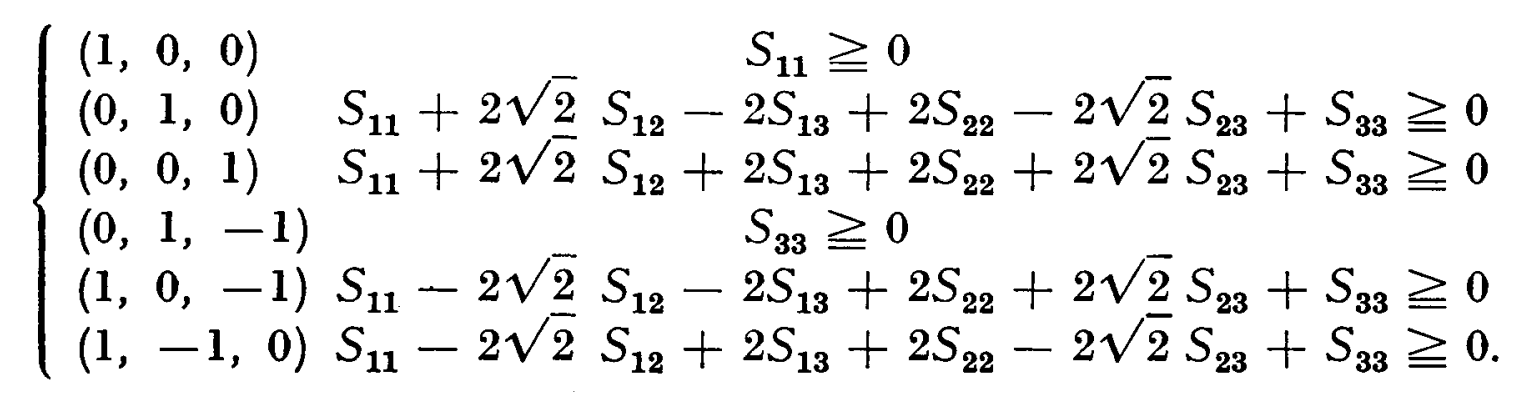

These inequalities determine a convex cone $C$ in the six-dimensional strain space of the components of $\boldsymbol{S}$, and the one-dimensional edge opposite the five-dimensional face $S_{11}=0$ is the half-line from the origin through the point:-

$$
S^{\dagger}=\left(\begin{array}{rrr}
2 & 0 & 0 \\
0 & -1 & 0 \\
0 & 0 & 0
\end{array}\right) .
$$

Our task is now, if we are given a stress, to decide whether any one of the possible rotations of that stress is capable of inducing any one of the geometrically possible strains. First we must study the geometry of the six-dimensional strain space.

LEMma 1. Consider the group of all matrices $\boldsymbol{X}$ such that the mapping $\boldsymbol{n}$ to $\boldsymbol{X} \boldsymbol{n}$ is a permutation of the twelve lattice points given above (formula 1.1). It is a transitive group over the twelve points, i.e. if $m$ and $n$ are any two then there is $\boldsymbol{X}$ in the group such that $\boldsymbol{m}=\boldsymbol{X n}$. 
Proof. The group contains all the six three-point matrices that permute the coordinate axes and also contains the negative of the unit matrix. The subgroup generated by these is transitive over all the six lattice points that have two coordinates zero, and is also transitive over the other six.

Also a straightforward calculation shows that the group contains the matrix:-

$$
\left(\begin{array}{rrr}
0 & 1 & 0 \\
1 & 0 & 0 \\
-1 & -1 & -1
\end{array}\right)
$$

which interchanges $(1,0,0)$ with $(0,1,-1)$. Therefore the group is transitive over all the twelve lattice points. Lemma 1 is a special case of a result given by Voronoï (page 152 of [1]). For this reference I am indebted to E. S. Barnes.

LEMma 2. If $\boldsymbol{A}$ and $\boldsymbol{B}$ are symmetric and if $\boldsymbol{n}^{\prime} \boldsymbol{A n}=\boldsymbol{n}^{\prime} \boldsymbol{B n}$ for each of the twelve lattice points of formula (1.1) then $\boldsymbol{A}=\boldsymbol{B}$.

Proof. Let $\boldsymbol{A}-\boldsymbol{B}=\boldsymbol{C}$, then by giving $\boldsymbol{n}^{\prime}$ successively the values $(1,0,0)$, $(0,1,0)$ and $(0,0,1)$ we see that the diagonal components of $C$ are all 0 , then the other values, $(1,-1,0)$ etc. show that the non-diagonal elements are also zero.

LEMma 3. If $\boldsymbol{X}$ is in the group of Lemma 1 then $\boldsymbol{R}=\boldsymbol{P} \boldsymbol{X} \boldsymbol{P}^{-1}$ is orthogonal.

Proof. $\boldsymbol{X}^{\prime} \boldsymbol{P}^{\prime} \boldsymbol{P} \boldsymbol{X}=\boldsymbol{P}^{\prime} \boldsymbol{P}$ by Lemma 2 .

Substituting $\boldsymbol{R} \boldsymbol{P}$ for $\boldsymbol{P} \boldsymbol{X}$ this gives

$$
\boldsymbol{P}^{\prime} \boldsymbol{R}^{\prime} \boldsymbol{R P}=\boldsymbol{P}^{\prime} \boldsymbol{P}
$$

Consequently $\boldsymbol{R}^{\prime} \boldsymbol{R}=\mathbf{1}$.

LEMMA 4. Given any one of the six inequalities (1.2), an orthogonal matrix $\boldsymbol{R}$ can be chosen such that the transformation of $\boldsymbol{S}$ to $\boldsymbol{R}^{\prime} \boldsymbol{S} \boldsymbol{R}$ permutes the inequalities among themselves and transforms the first into the given one.

Proof. First choose a matrix $\boldsymbol{X}$ of the group of Lemma 1 that gives an appropriate transformation of the lattice points (see 1.2), put $\boldsymbol{R}=\boldsymbol{P} \boldsymbol{X} \boldsymbol{P}^{-1}$.

Then if $\boldsymbol{X n}=\boldsymbol{m}$ the inequality

is transformed to

$$
\boldsymbol{n}^{\prime} \boldsymbol{P}^{\prime} \boldsymbol{S P n} \geqq 0
$$

$$
n^{\prime} P^{\prime} R^{\prime} S R P n \geqq 0
$$

or

$$
\boldsymbol{n}^{\prime} \boldsymbol{X}^{\prime} \boldsymbol{P}^{\prime} \boldsymbol{S P X n} \geqq 0
$$

or

$$
\boldsymbol{m}^{\prime} \boldsymbol{P}^{\prime} \mathbf{S P} \boldsymbol{m} \geqq 0
$$


The matrix $S^{\dagger}$ (1.3) shows that of the six linear forms in the strain space given in (1.2) the first is linearly independent of the others, and by Lemma 4 it follows that all are linearly independent.

Now to go back to our strength criterion; the sand will collapse under a stress $\boldsymbol{F}$ if for some rotation $\boldsymbol{F}^{*}=\boldsymbol{R}^{\prime} \boldsymbol{F R}$ of $\boldsymbol{F}$ and some $\boldsymbol{S}^{*}$ in $C$ (i.e. any geometrically possible strain) Spur $F^{*} S^{*}>0$ or in tensor notation $\Sigma F_{i j}^{*} S_{i j}^{*}>0$, i.e. the virtual work is positive. (We take tensile stress as positive.)

The first step in simplifying this is to show that we need only consider $S^{*}$ in the edges of $C$. To show this take the six linear forms of (1.2) as coordinates $\left(x_{1}, \cdots x_{6}\right)$ in the strain space; the virtual work is then a linear form, say $\Sigma a_{i} x_{i}$. The condition on the $a_{i}$ : ' $\Sigma a_{i} x_{i}>0$ for some $x_{1}, \cdots x_{6}$, all $\geqq 0$ ' is equivalent to 'Some $a_{i}>0$ ' or ' $\Sigma a_{i} x_{i}>0$ for some coordinate positive and the others all zero'.

The second step in simplifying the criterion is to show that instead of considering all $S^{*}$ in all the six edges of $C$ we need only consider the strain $S^{\dagger}(1.3)$.

To show this suppose that $F$ is such that there exists an orthogonal matrix $R$ and a strain $S^{*}$ on one edge of $C$ with Spur $R^{\prime} F R S^{*}>0$. By Lemma 4 there is an orthogonal matrix $Q$ and a positive scalar $q$ such that $q \boldsymbol{S}^{*}=\boldsymbol{Q}^{\prime} \boldsymbol{S}^{\dagger} \boldsymbol{Q}$; also $\boldsymbol{T}=\boldsymbol{R} \boldsymbol{Q}^{\prime}$ is orthogonal and:-

$$
\begin{aligned}
& \text { Spur } \boldsymbol{T}^{\prime} \boldsymbol{F} \boldsymbol{T} \boldsymbol{S}^{\dagger} \\
= & \operatorname{Spur} \boldsymbol{Q}^{\prime} \boldsymbol{T}^{\prime} \boldsymbol{F} \boldsymbol{T} \boldsymbol{S}^{\dagger} \boldsymbol{Q} \\
= & \operatorname{Spur} \boldsymbol{R}^{\prime} \boldsymbol{F} \boldsymbol{R} \boldsymbol{Q}^{\prime} \boldsymbol{S}^{\dagger} \boldsymbol{Q} \\
= & q \text { Spur } \boldsymbol{R}^{\prime} \boldsymbol{F} \boldsymbol{R}^{*}>0 .
\end{aligned}
$$

The criterion can now be expressed as 'A stress $\boldsymbol{F}$ will cause collapse of the sand if there is an orthogonal matrix $\boldsymbol{T}$ such that Spur $\boldsymbol{T}^{\prime} \boldsymbol{F T S} \boldsymbol{T}^{\dagger}>0$.'

Now $\boldsymbol{F}$ can be made diagonal by a further orthogonal transformation, so in the criterion above we can suppose that it is diagonal, therefore put:-

$$
\boldsymbol{F}=\left(\begin{array}{rrr}
-a & 0 & 0 \\
0 & -b & 0 \\
0 & 0 & -c
\end{array}\right) \quad \boldsymbol{T}=\left(\begin{array}{lll}
l_{1} & m_{1} & n_{1} \\
l_{2} & m_{2} & n_{2} \\
l_{3} & m_{3} & n_{3}
\end{array}\right)
$$

We can now express the result as that the sand will remain rigid under a stress with eigenvalues $-a,-b$, and $-c$ if for any two sets of perpendicular direction cosines $\left(l_{1}, m_{1}, n_{1}\right)$ and $\left(l_{2}, m_{2}, n_{2}\right):-$

$$
2\left(a l_{1}^{2}+b m_{1}^{2}+c n_{1}^{2}\right) \geqq\left(a l_{2}^{2}+b m_{2}^{2}+c n_{2}^{2}\right) .
$$

This implies that $4\left(a l_{1}^{2}++\right) \geqq 2\left(a l_{2}^{2}++\right) \geqq\left(a l_{1}^{2}++\right)$ and therefore that $a, b$ and $c$ are all strictly positive. (We ignore the case of all zero).

Now $a l_{1}^{2}+b m_{1}^{2}+c n_{1}^{2}$ and $a l_{2}^{2}+b m_{2}^{2}+c n_{2}^{2}$ are the squares of the lengths of two conjugate semi-diameters of the ellipsoid:- 


$$
\frac{x^{2}}{a}+\frac{y^{2}}{b}+\frac{z^{2}}{c}=1
$$

and the condition for rigidity is therefore that no semi-diameter should exceed $\sqrt{ } \mathbf{2}$ times any conjugate semi-diameter. The ratio of two conjugate semi-diameters can (by continuity) take any value between the least and greatest of the six ratios of the semi-axes, so the condition for rigidity is that none of the six ratios $a / b$ etc. should exceed two.

Our conclusion therefore is that the frictionless model of sand will withstand a stress if and only if the three principal stresses are all negative (i.e. compressive) and the largest of them is less than twice the smallest.

\section{The Effect of Friction}

Suppose that the coefficient of friction between any two sand grains is $\mu$. The assumptions and reasoning in this section are not valid except to first order in $\mu$, but in order to give the reader an insight into the behaviour of sand for larger values of $\mu$, the calculations are exact.

Take coordinates as before. In the case of no friction we found that the collapse can be assumed to be towards the strain $S^{\dagger}(1.3)$ and that the critical stress (that just suffices to cause collapse) has its principal axes the same as those of strain, the $z$-axis being that of the intermediate of the three principal stresses.

We make the assumptions that when there is friction the sand will collapse in the same way, and that the critical stress has the $z$-axis as the principal direction belonging to the intermediate of the three eigenvalues, but we leave the directions of the other prinicpal axes to be determined later.

The first task is to relate the forces on one sand grain to the mean stress tensor of the sand; to do this it is enough (since we deal only with ratios of forces) to consider the mean stress inside one grain, or the volumeintegral of the stress tensor through one grain of sand. Suppose that a body exerts a number of forces on its surroundings, the $k$ th being a force $p_{k i}$ at a point $x_{k i}$; then the integral of the stress tensor through the body is:-

$$
-\Sigma p_{k i} x_{k j} \text {. }
$$

The five normal reactions exerted by the hemisphere $z>0$ are given below, the first column giving the magnitudes $(p, q, \cdots)$ and the second the direction cosines of the line of action, which multiplied by $d / 2$ give the coordinates of the points $(P, Q, \cdots)$ of application.

$\begin{array}{lrr}p\left(-\frac{1}{2},\right. & -\sqrt{ } \frac{1}{2}, & \left.\frac{1}{2}\right) \\ q\left(-\frac{1}{2},\right. & \sqrt{ } \frac{1}{2}, & \left.\frac{1}{2}\right) \\ r\left(\frac{1}{2},\right. & -\sqrt{ } \frac{1}{2}, & \left.\frac{1}{2}\right) \\ s\left(\frac{1}{2},\right. & \sqrt{ } \frac{1}{2}, & \left.\frac{1}{2}\right) \\ t(0, & 0, & 1) .\end{array}$


They are balanced by equal forces at the diametrically opposite points $\left(P^{\prime}, Q^{\prime}, \cdots\right)$. The contribution to the integral of the stress tensor from these ten forces is:-

$$
-d\left(\begin{array}{ccc}
\frac{p+q+r+s}{4} & \frac{p-q-r+s}{2 \sqrt{ } 2} & \frac{-p-q+r+s}{4} \\
\frac{p-q-r+s}{2 \sqrt{ } 2} & \frac{p+q+r+s}{2} & \frac{-p+q-r+s}{2 \sqrt{ } 2} \\
\frac{-p-q+r+s}{4} & \frac{-p+q-r+s}{2 \sqrt{ } 2} & \frac{p+q+r+s}{4}+t
\end{array}\right)
$$

We assume that the (1.3) and (2.3) components are zero and we may therefore take $p=s=1+\varphi / \sqrt{ } 2$ and $q=r=1-\varphi / \sqrt{ } 2$ where we may take $\varphi \geqq 0$. (To first order, $\varphi$ is the angle between the principal axes of stress and strain).

Now to consider the frictional forces. The strain $\boldsymbol{S}^{\dagger}$ has no effect at $T$ or $T^{\prime}$, at $P, P^{\prime}, S$ and $S^{\prime}$ it tends to cause rotation of the sphere about the $z$-axis, while at $Q, Q^{\prime}, R$ and $R^{\prime}$ it tends to cause the opposite rotation. As $p$ and $s$ are larger than $q$ and $r$ the rotation will be such that there is no slipping at $P, P^{\prime}, S$ or $S^{\prime}$ and all the frictional forces are equal to $\mu q=\mu r=\mu(1-\varphi / \sqrt{ } 2)$ and are perpendicular to the $z$-axis. The frictional forces exerted by the upper hemisphere are therefore as follows, the first column giving the direction of the force and the second giving the point of application:

$$
\begin{array}{rrrr}
\sqrt{2 / 3}, & -\sqrt{1 / 3}, & 0) & P \\
\sqrt{2 / 3}, & \sqrt{1 / 3}, & 0) & Q \\
(-\sqrt{2 / 3}, & -\sqrt{1 / 3}, & 0) & R \\
(-\sqrt{2 / 3}, & \sqrt{1 / 3}, & 0) & S .
\end{array}
$$

The total contribution to the integral of the stress tensor from the ten normal and eight tangential forces is a negative multiple of

$$
\left(\begin{array}{ccc}
\frac{\sqrt{ } 3-2 \mu \sqrt{ } 2+2 \mu \varphi}{\sqrt{ } 3} & \varphi & 0 \\
\varphi & \frac{2 \sqrt{ } 3+2 \mu \sqrt{ } 2-2 \mu \varphi}{\sqrt{ } 3} & 0 \\
0 & 0 & 1+t
\end{array}\right)
$$

Consider the two eigenvalues other than $1+t$, the ratio of their sum to their difference is:-

$$
3\left[(1+4 \mu \sqrt{2 / 3}-4 \mu \varphi / \sqrt{ } 3)^{2}+4 \varphi^{2}\right]^{-1 / 2}
$$


which as $\varphi$ varies reaches its maximum of:-

$$
\frac{3 \sqrt{1+4 \mu^{2} / 3}}{1+4 \mu \sqrt{2 / 3}} \text { when } \varphi=\frac{\sqrt{ } 3 \mu+4 \sqrt{ } 2 \mu^{2}}{3+4 \mu^{2}} .
$$

(Here we must have $\mu<\sqrt{ } 6$ to make $1-\varphi / \sqrt{ } 2$ positive).

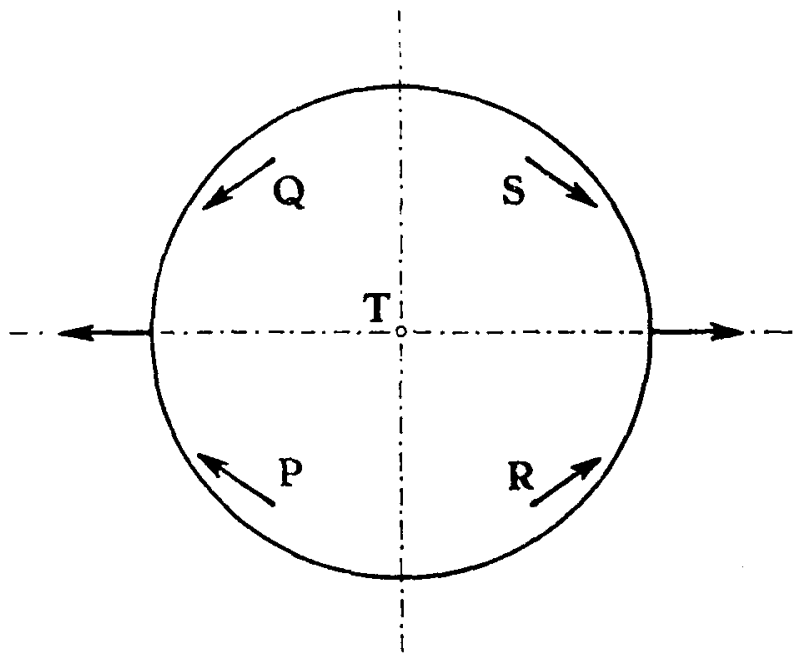

Fig. 1. Projection on the $x y$ plane showing the directions of initial motion of the neighbouring spheres and their points of contact.

As $\varphi$ varies, therefore, the ratio of the largest to the smallest principal stress has a minimum of:-

$$
\frac{3 \sqrt{1+4 \mu^{2} / 3}+1+4 \mu \sqrt{2 / 3}}{3 \sqrt{1+4 \mu^{2} / 3}-1-4 \mu \sqrt{2 / 3}}
$$

or when expanded:

$$
2+2 \mu \sqrt{ } 6+7 \mu^{2}-8 \sqrt{ } 6 \mu^{3} / 9+\cdots
$$

At this stage we may examine again the validity of our assumptions about the effect of friction.

In $\S 1$ we saw that with no friction the orientation of the lattice that is most favourable to collapse is with the principal directions of strain the same as those of stress. The metaphysical principle of continuity leads us to suppose that when the coefficient of friction is small the directions will be nearly the same.

The assumptions that the principal strains will be in the ratio $2:-1: 0$ and that the principal direction of zero strain is that of the intermediate of the three principal stresses are therefore supposed to be valid approximations for small friction. That the assumptions are not otherwise valid is shown by two features of the analysis above. Firstly we supposed that $1+t$ is the intermediate of the three eigenvalues of the matrix above (2.1) which is true only if $\mu$ is less than some quantity depending on $t$, i.e. on the intermediate of the three principal stresses. Secondly we have seen that the 
orientation of the lattice so that the principal axes of stress and strain coincide is not the most favourable to collapse, but that a more favourable one can be found by rotating the lattice about an axis in the direction of the intermediate of the three principal stresses; a rotation about some other axis might give an even more favourable orientation.

Thus our criterion for rigidity is that the principal stresses be compressive and the ratio of the largest to the smallest principal stress should be less than:

$$
2+2 \mu \sqrt{ } 6+\text { Unknown higher powers of } \mu .
$$

Even if the higher coefficients were known and gave a convergent power series in $\mu$, the range of validity of this result would depend on the intermediate of the three principal stresses. This intermediate eigenvalue determines the $t$ of the matrix (2.1), and this has to be positive to make the reasoning of this section valid. Thus the range of validity of (2.3) will be small if the two smaller of the principal stresses are nearly equal.

\section{Empirical Results and the Effect of Wetness}

In this section we change the sign of the stress tensor to adopt the usual convention in soil mechanics that compressive stresses are positive. Among engineers it is generally supposed that the condition for a stress to cause rupture of any kind of soil is given by Coulomb's equation, which may be written:

$$
S_{1}=2 c \tan \left(45^{\circ}+\frac{1}{2} \varphi\right)+S_{3} \tan ^{2}\left(45^{\circ}+\frac{1}{2} \varphi\right)
$$

where $S_{1}$ and $S_{3}$ are the largest and smallest of the principal stresses and $c$ and $\varphi$ are empirical constants, called respectively the 'cohesion' and the 'angle of shearing resistance' or 'angle of internal friction' (See, for example, K. Terzaghi, [2]). For dry sand $c=0$ and the observed values of $\varphi$ are from $30^{\circ}$ to $50^{\circ}$ ([2] page 8$)$, giving the critical ratio of principal stresses $\left(\tan ^{2}\left(45^{\circ}+\frac{1}{2} \varphi\right)\right)$ from 3 to 7.5 .

For wet sand the observed value of $c$ and $\varphi$ depend on the water content, the way that the stress has varied with time and percolation of water in and out of the sand. These phenomena are illustrated by walking on a sandy beach a little way up from the sea, and the qualitative explanation is well known, that distortion of the sand packing and capillarity reduce the pressure of the water in the interstices to below atmospheric, thereby tending to pull the sand grains together. ([2] Chapter XIV).

In order to fit these phenomena into the theory we must first see what a stress tensor is. The difficulty is that it is arbitrary to the extent of adding any uniform tensor (i.e. one whose Cartesian components are in- 
dependent of position); for stresses are recognizable only by their space derivatives and discontinuities.

In engineering it is usually convenient to take the atmosphere as under zero stress. In this convention let the matrix $\boldsymbol{\sigma}$ represent the stress tensor and let $\boldsymbol{F}$ be minus the stress tensor that was considered in $\S \mathbf{1}$ and $\S 2$, made up of the forces exerted by the sand grains on one another at their points of contact.

If the space between the grains is filled with water at a pressure $k$ below atmospheric then:

$$
\boldsymbol{F}=\boldsymbol{G}+k \mathbf{1} \text { (where } \mathbf{1} \text { is the unit matrix). }
$$

If $S_{1}$ and $S_{3}$ are the greatest and least eigenvalues of $G$ then our criterion for the rigidity of the sand becomes:

$$
0<S_{1}+k<\left(S_{3}+k\right)(2+2 \mu \sqrt{ } 6)
$$

If the sand is wet but not saturated a similar effect occurs, because of a small amount of water retained round each point of contact between the sand grains ([2] page 301).

\section{References}

[1] Voronoï, G., „Sur quelques propriétés des formes quadratiques positives parfaites" $J$. reine angew. Math., 133 (1907), 97-178.

[2] Terzaghi, K., Theoretical Soil Mechanics, Wiley, New York, (1943). 